Теорія Ймовір. та Матем. Статист. Вип. 74, 2006
Theor. Probability and Math. Statist.

No. 74, 2007, Pages 29-35

S 0094-9000(07)00695-3

Article electronically published on June 25, 2007

\title{
WIDE SENSE STATIONARY SOLUTIONS OF DIFFERENCE EQUATIONS IN A BANACH SPACE
}

UDC 519.21

\author{
M. F. GORODNII
}

\begin{abstract}
A criterion is proved for the existence of a unique wide sense stationary solution of a linear difference equation with operator coefficients in a Banach space. The stability of this solution with respect to small perturbations of operator coefficients is proved.
\end{abstract}

Let $(\Omega, F, \mathrm{P})$ be a complete probability space, $\left(X,\|\cdot\|_{X}\right)$ a complex separable Banach space with zero element $0_{X}$ and $\sigma$-algebra of Borel sets $\mathcal{B}(X), L(X)$ a Banach space of all linear bounded operators acting from $X$ to $X, I_{X}$ and $O_{X}$ the unit and zero operators in $X$, respectively. We denote the norm of an operator $B \in L(X)$ by $\|B\|_{L(X)}$.

Definition 1. A sequence of $X$-valued random elements $\eta:=\left\{\eta_{n}: n \in \mathbf{Z}\right\}$ defined on the probability space $(\Omega, F, \mathrm{P})$ is called stationary (in a wide sense) if

1) $\sup _{n \in \mathbf{Z}} \mathrm{E}\left\|\eta_{n}\right\|_{X}^{2}<+\infty$

2) $\mathrm{E} \eta_{n}=\mathrm{E} \eta_{0}$ for all $n \in \mathbf{Z}$,

3) for all $n, k \in \mathbf{Z}$ and all $x^{*}, y^{*} \in X^{*}$

$$
\operatorname{cov}\left(\left\langle\eta_{n+k}, x^{*}\right\rangle,\left\langle\eta_{k}, y^{*}\right\rangle\right)=\operatorname{cov}\left(\left\langle\eta_{n}, x^{*}\right\rangle,\left\langle\eta_{0}, y^{*}\right\rangle\right) .
$$

Here $X^{*}$ is the dual space to the Banach space $X$ and $\left\langle x, x^{*}\right\rangle$ denotes the action of the functional $x^{*} \in X^{*}$ at the element $x \in X$.

Remark 1 . It is shown in (1) that conditions 2) and 3) in the definition of a stationary sequence do not necessarily imply condition 1) for the case of an infinite-dimensional Banach space.

Let $\eta$ be a stationary sequence of $X$-valued random elements, let an operator

$$
A: D \subset X \rightarrow X
$$

be closed, and let $\left\{A_{n}: n \in \mathbf{Z}\right\}$ be a sequence of operators in $L(X)$ such that

$$
\sum_{n \in \mathbf{Z}}\left\|A_{n}\right\|_{L(X)}<+\infty
$$

Definition 2. A sequence of $X$-valued random elements

$$
\xi:=\left\{\xi_{n}: n \in \mathbf{Z}\right\}
$$

defined on a probability space $(\Omega, F, \mathrm{P})$ is called a stationary solution of the difference equation

$$
A \xi_{n}=\sum_{k \in \mathbf{Z}} A_{k} \xi_{n+k}+\eta_{n}, \quad n \in \mathbf{Z}
$$

2000 Mathematics Subject Classification. Primary 60G10, 39A10; Secondary 47A50.

Key words and phrases. Difference equation, operator coefficients, wide sense stationary solutions.

(C)2007 American Mathematical Society 
if equality (2) holds, $\xi$ is a stationary sequence, and for every $n \in \mathbf{Z}$,

$$
\xi_{n}: \Omega \rightarrow D
$$

with probability 1.

The uniqueness of a solution of equation (2) is understood in the sense of the stochastic equivalence.

The aim of this paper is to obtain a criterion for the existence of a unique stationary solution of equation (2) and to study its stability with respect to small perturbations of the operator coefficients.

\section{Stationary solutions of Difference Equation (2)}

Put

$$
S:=\{z \in \mathbf{C}:|z|=1\}, \quad f(z):=\sum_{n \in \mathbf{Z}} A_{n} z^{n}, \quad z \in S .
$$

An answer to the question on whether or not a unique stationary solution of equation (2) exists is given in the following result.

Theorem 1. The following two statements are equivalent:

$i_{1}$ ) Difference equation (2) has a unique stationary solution $\xi$ for an arbitrary stationary sequence $\eta$.

$\left.i_{2}\right)$ For every $z \in S, A-f(z)$ has the inverse operator

$$
(A-f(z))^{-1} \in L(X) .
$$

Remark 2. If a sequence $\left\{A_{n}: n \in \mathbf{Z}\right\}$ satisfies a stronger assumption than (1), namely if $f$ is an analytic function in a certain ring containing $S$, then a criterion for the existence of a unique stationary (in a restricted sense) solution and sufficient conditions for the existence of a stationary solution of equation (2) are given in [1].

We need the following auxiliary results for the proof of Theorem 1.

Lemma 1. If assumption $i_{2}$ ) holds, then the nonrandom difference equation

$$
A x_{n}=\sum_{k \in \mathbf{Z}} A_{k} x_{n+k}+y_{n}, \quad n \in \mathbf{Z},
$$

has a unique bounded in the norm of $X$ solution $x:=\left\{x_{n}: n \in \mathbf{Z}\right\}$ for an arbitrary bounded sequence $y:=\left\{y_{n}: n \in \mathbf{Z}\right\}$.

Proof. Note that $D$ is a complex Banach space with the norm

$$
\|u\|_{D}:=\|u\|_{X}+\|A u\|_{X}, \quad u \in D .
$$

We also note that any bounded solution $x$ of difference equation (3) corresponding to a $\left(X,\|\cdot\|_{X}\right)$ bounded sequence $y$ is a $\left(D,\|\cdot\|_{D}\right)$ bounded sequence.

Denote by $L(X, D)$ the set of all linear bounded operators acting from $X$ to $D$. Put $T:=(A-f(1))^{-1}$. It follows from condition $\left.i_{2}\right)$ that

$$
T: X \rightarrow D, \quad T \in L(X) .
$$

It is easy to check that $T \in L(X, D), T A \in L(D)$, the operator $T A_{n}: D \rightarrow D$ belongs to $L(D)$ for any $n \in \mathbf{Z}$, and

$$
\left\|T A_{n}\right\|_{L(D)} \leq\left\|A_{n}\right\|_{L(X)}\|T\|_{L(X, D)} .
$$

Thus

$$
\|T A\|_{L(D)}+\sum_{n \in \mathbf{Z}}\left\|T A_{n}\right\|_{L(D)}<+\infty
$$


in view of condition (1). Note also that

$$
(A-f(z)) \in L(D, X), \quad(A-f(z))^{-1} \in L(X, D),
$$

for all $z \in S$ and therefore the operator

$$
\left(T A-\sum_{n \in \mathbf{Z}} T A_{n} z^{n}\right): D \rightarrow D
$$

belongs to $L(D)$ and has the continuous inverse operator

$$
(A-f(z))^{-1} T^{-1}
$$

for all $n \in \mathbf{Z}$ according to condition $i_{2}$ ).

The generalization of the Wiener theorem on the absolutely convergent Fourier series proved by Bochner and Phillips 2] and condition (5) imply that there exists a sequence of operators

$$
\left\{C_{n}: n \in \mathbf{Z}\right\} \subset L(D)
$$

such that

$$
\begin{gathered}
\sum_{n \in \mathbf{Z}}\left\|C_{n}\right\|_{L(D)}<+\infty, \\
\text { for all } z \in S, \quad(A-f(z))^{-1} T^{-1}=\sum_{n \in \mathbf{Z}} C_{n} z^{n} .
\end{gathered}
$$

Since the operator $T$ belongs to the space $L(X, D)$ and is continuously invertible, the mapping

$$
X \supset\left\{x_{n}: n \in \mathbf{Z}\right\} \rightarrow\left\{T x_{n}: n \in \mathbf{Z}\right\} \subset D
$$

is a bijection between the sets of all bounded sequences in the spaces $\left(X,\|\cdot\|_{X}\right)$ and $\left(D,\|\cdot\|_{D}\right)$. Thus Lemma 1 holds if and only if the difference equation

$$
T A u_{n}=\sum_{k \in \mathbf{Z}} T A_{k} u_{n+k}+v_{n}, \quad n \in \mathbf{Z},
$$

has a unique bounded solution $u:=\left\{u_{n}: n \in \mathbf{Z}\right\}$ for an arbitrary bounded in $D$ sequence $v:=\left\{v_{n}: n \in \mathbf{Z}\right\}$.

Further, using properties of the inverse operator, we derive from relations (6) and (7) that

$$
\begin{gathered}
(T A) C_{m}-\sum_{k \in \mathbf{Z}}\left(T A_{k}\right) C_{m+k}=C_{m}(T A)-\sum_{k \in \mathbf{Z}} C_{m+k}\left(T A_{k}\right), \\
(T A) C_{-m}=\sum_{k \in \mathbf{Z}}\left(T A_{k}\right) C_{-m+k}+\delta_{m, 0} I_{D}
\end{gathered}
$$

for all $m \in \mathbf{Z}$. Here $\delta_{m, 0}=0$ for $m \neq 0, \delta_{0,0}=1$, and $I_{D}$ is the unit operator in $D$. Following the method used in the proof of the implication ii) $\Rightarrow$ iii) in Theorem 1 of the paper [3], one can check that there exists a unique bounded solution $u$ of equation (8) for an arbitrary bounded in $D$ sequence $v$ and moreover

$$
u_{n}=\sum_{k \in \mathbf{Z}} C_{n-k} v_{k}, \quad n \in \mathbf{Z} .
$$

Lemma 1 is proved.

Remark 3. Lemma 1 also holds for a nonseparable space $X$.

As usual, a sequence $\eta$ of $X$-valued random elements is called mean square bounded if it satisfies condition 1) of Definition 1. The uniqueness of a mean square bounded solution $\xi$ of equation (2) is defined similarly to Definition 2. 
M. F. GORODNII

Lemma 2. Assume that condition $\left.i_{2}\right)$ of Theorem 1 holds. Then the difference equation (2) has a unique mean square bounded solution $\xi$ for an arbitrary mean square bounded sequence $\eta$.

Proof. Let $Y:=L_{2}(\Omega, X)$ (see, for example, 4]). The norm in the space $Y$ is denoted by $\|\cdot\|_{Y}$. Note that $D \in \mathcal{B}(X)$ and $A \eta$ is a random element if an $X$-valued random element $\eta$ acts from $\Omega$ to $D$.

Put

$$
\tilde{D}:=\{\eta \in Y \mid \eta: \Omega \rightarrow D, A \eta \in Y\}
$$

and define the operator $\tilde{A}: \tilde{D} \rightarrow Y$ by

$$
\text { for all } \eta \in \tilde{D}, \quad(\tilde{A} \eta)(\omega):=A \eta(\omega), \quad \omega \in \Omega .
$$

Denote by $\rho(A)$ the resolvent of the operator $A$. Following the idea of the proof of Lemma 2 of [1] one can check that

$\left.j_{1}\right) \tilde{A}$ is a closed operator, $\rho(\tilde{A})=\rho(A)$,

$\left.j_{2}\right)$ if $\lambda \in \rho(A)$, then $\left(\tilde{A}-\lambda I_{Y}\right)^{-1}=\left(\left(A-\lambda I_{X}\right)^{-1}\right)^{\sim}$,

$\left.j_{3}\right)$ if additionally $A \in L(X)$, then $\tilde{D}=Y, \tilde{A} \in L(Y)$, and $\|\tilde{A}\|_{L(Y)}=\|A\|_{L(X)}$.

Therefore Lemma 2 holds if and only if the nonrandom difference equation

$$
\tilde{A} \xi_{n}=\sum_{k \in \mathbf{Z}} \tilde{A}_{k} \xi_{n+k}+\eta_{n}, \quad n \in \mathbf{Z}
$$

has a unique bounded solution $\xi$ for an arbitrary bounded in $Y$ sequence $\eta$. The latter condition holds in view of Lemma 1 and properties $\left.j_{1}\right)-j_{3}$ ). Moreover, according to equality (9) the unique mean square bounded solution $\xi$ of equation (2) corresponding to a mean square bounded sequence $\eta$ is represented as

$$
\xi_{n}=\sum_{k \in \mathbf{Z}} C_{n-k} \eta_{k}, \quad n \in \mathbf{Z} .
$$

Lemma 2 is proved.

Proof of Theorem 1. First we prove that $\left.i_{1}\right) \Rightarrow i_{2}$ ). Fix $z \in S, u \in X$, and $u \neq 0_{X}$. For $\eta_{0}$ we take a random element such that

$$
\mathrm{P}\left\{\eta_{0}=u\right\}=\mathrm{P}\left\{\eta_{0}=-u\right\}=\frac{1}{2} .
$$

Put $\eta_{n}:=\eta_{0} z^{n}, n \in \mathbf{Z}$. It is easy to check that the sequence $\left\{\eta_{n}: n \in \mathbf{Z}\right\}$ is stationary. According to condition $i_{1}$ ), equation (2) for this sequence has a unique stationary solution $\xi$. Thus

$$
A \xi_{n} z^{-n}=\sum_{k \in \mathbf{Z}} A_{k} z^{k}\left(\xi_{n+k} z^{-n-k}\right)+\eta_{0}, \quad n \in \mathbf{Z}
$$

with probability one, whence

$$
A\left(\xi_{n+1} z^{-n-1}-\xi_{n} z^{-n}\right)=\sum_{k \in \mathbf{Z}} A_{k} z^{k}\left(\xi_{n+k+1} z^{-n-k-1}-\xi_{n+k} z^{-n-k}\right), \quad n \in \mathbf{Z}
$$

with probability one.

Condition $i_{1}$ ) means in particular that the homogeneous difference equation (2) has a unique zero (stationary) solution. Hence (12) implies that

$$
\xi_{n+1} z^{-n-1}=\xi_{0}, \quad n \in \mathbf{Z},
$$

with probability one. Using (11), we prove that $(A-f(z)) \xi_{0}=\eta_{0}$ with probability one. 
Therefore we proved that the equation $(A-f(z)) w=u$ has a solution $w$ for an arbitrary $u \in X$. Were this solution not unique, one would find an element $v \in X$, $v \neq 0_{X}$, such that

$$
(A-f(z)) v=0_{X} .
$$

Then the homogeneous difference equation (2) has a nonzero stationary solution

$$
\zeta_{n}=\zeta_{0} z^{n}
$$

where $\zeta_{0}$ is a random element such that

$$
\mathrm{P}\left\{\zeta_{0}=v\right\}=\mathrm{P}\left\{\zeta_{0}=-v\right\}=\frac{1}{2} .
$$

The Banach inverse operator theorem for a closed operator implies that the operator

$$
(A-f(z))^{-1} \in L(X)
$$

exists.

Now we prove the implication $\left.i_{2}\right) \Rightarrow i_{1}$ ). If condition $i_{2}$ ) holds, then Lemma 2 yields that equation (2) for a fixed stationary sequence $\eta$ has a unique mean square bounded solution $\xi$ that can be represented in the form of series (10). Using representation (10), one can easily check that this solution is stationary.

Theorem 1 is proved.

\section{The stability of Stationary solutions of the Difference equation $(2)$}

In this section, we study the stability of stationary solutions of equation (2) with respect to small perturbations of the operator coefficients. This is an important question from the point of view of applications where the structure of perturbations is not known completely and is modelled by a difference equation.

The following theorem contains the main result in this direction.

Theorem 2. Assume that condition $\left.i_{2}\right)$ of Theorem 1 holds. Let $T_{n}(m), T_{n, k}(m), n \in \mathbf{Z}$, $k \in \mathbf{Z}, m \in \mathbf{N}$, be a family of operators of $L(X)$ such that

$$
\delta_{m}:=\sup _{n \in \mathbf{Z}}\left(\left\|T_{n}(m)-T_{n, 0}(m)\right\|_{L(X)}+\sum_{k \in \mathbf{Z}, k \neq 0}\left\|T_{n, k}(m)\right\|_{L(X)}\right) \rightarrow 0, \quad m \rightarrow \infty .
$$

Then

$\left.a_{1}\right)$ for all stationary sequences $\eta$ and for all sufficiently large numbers $m$, the difference equation

$$
\left(A+T_{n}(m)\right) \xi_{n}(m)=\sum_{k \in \mathbf{Z}}\left(A_{k}+T_{n, k}(m)\right) \xi_{n+k}(m)+\eta_{n}, \quad n \in \mathbf{Z},
$$

has a unique mean square bounded solution

$$
\xi(m):=\left\{\xi_{n}(m): n \in \mathbf{Z}\right\} .
$$

$\left.a_{2}\right)$ Also,

$$
\sup _{n \in \mathbf{Z}} \mathrm{E}\left\|\xi_{n}(m)-\xi_{n}\right\|_{X}^{2}=O\left(\delta_{m}^{2}\right), \quad m \rightarrow \infty
$$

where $\xi:=\left\{\xi_{n}: n \in \mathbf{Z}\right\}$ is a unique stationary solution of equation (2) corresponding to the sequence $\eta$. 
M. F. GORODNII

Proof. We use notation introduced in the proof of Lemmas 1 and 2. Similarly to $\left(D,\|\cdot\|_{D}\right)$ we construct the Banach space $\left(\tilde{D},\|\cdot\|_{\tilde{D}}\right)$. Put

$$
W:=\left\{\zeta:=\left\{\zeta_{n}: n \in \mathbf{Z}\right\} \subset \tilde{D}:\|\zeta\|_{W}:=\sup _{n \in \mathbf{Z}}\left\|\zeta_{n}\right\|_{\tilde{D}}<+\infty\right\} .
$$

Then $\left(W,\|\cdot\|_{W}\right)$ is a complex Banach space equipped with the operations of addition defined coordinatewise and multiplication to scalars. Define the operators $G$ and $G_{m}$, $m \geq 1$, acting from $W$ in $W$ as follows:

$$
\begin{gathered}
G \zeta:=\left\{(G \zeta)_{n}=(T A)^{\sim} \zeta_{n}-\sum_{k \in \mathbf{Z}}\left(T A_{k}\right)^{\sim} \zeta_{n+k}: n \in \mathbf{Z}\right\}, \\
G_{m} \zeta:=\left\{\left(G_{m} \zeta\right)_{n}=\left(T\left(A+T_{n}(m)\right)\right)^{\sim} \zeta_{n}-\sum_{k \in \mathbf{Z}}\left(T\left(A_{k}+T_{n, k}(m)\right)\right)^{\sim} \zeta_{n+k}: n \in \mathbf{Z}\right\}, \\
\zeta \in W .
\end{gathered}
$$

It is easy to check that these operators belong to $L(W)$. Now we estimate $\left\|G-G_{m}\right\|_{L(W)}$. We have

since

$$
\left\|G-G_{m}\right\|_{L(W)} \leq\|T\|_{L(X, D)} \delta_{m},
$$

$$
\begin{aligned}
\left\|(G \zeta)_{n}-\left(G_{m} \zeta\right)_{n}\right\|_{\tilde{D}} & =\left\|\left(T T_{n, 0}(m)-T T_{n}(m)\right)^{\sim} \zeta_{n}+\sum_{k \in \mathbf{Z}, k \neq 0}\left(T T_{n, k}(m)\right)^{\sim} \zeta_{n+k}\right\|_{\tilde{D}} \\
& \leq\left(\left\|T T_{n, 0}(m)-T T_{n}(m)\right\|_{L(D)}+\sum_{k \in \mathbf{Z}, k \neq 0}\left\|T T_{n, k}(m)\right\|_{L(D)}\right)\|\zeta\|_{W} \\
& \leq\|T\|_{L(X, D)} \delta_{m}\|\zeta\|_{W}
\end{aligned}
$$

for all $n \in \mathbf{Z}$ in view of conditions $\left.j_{1}\right)-j_{3}$ ) and appropriate bounds for the norms similar to $(4)$.

It follows from Lemma 2 and the Banach inverse operator theorem that the inverse operator $G^{-1} \in L(W)$ exists. Put

$$
\gamma_{m}:=\left\|G^{-1}\right\|_{L(W)}\|T\|_{L(X, D)} \delta_{m}, \quad m \geq 1 .
$$

Since $\delta_{m} \rightarrow 0, m \rightarrow \infty$, one can claim that

$$
\text { there exists } m_{0} \in \mathbf{N} \text { such that } \gamma_{m}<1 \text { for all } m \geq m_{0} \text {. }
$$

Thus the operator $G_{m}$ has the inverse $G_{m}^{-1} \in L(W)$ for all $m \geq m_{0}$ (see [5]). Since the difference equation (13) is equivalent to the equation $G_{m} \xi=T \eta$ in the space $W$ where $T \eta:=\left\{T \eta_{n}: n \in \mathbf{Z}\right\}$, we conclude that condition $a_{1}$ ) holds for $m \geq m_{0}$.

Condition $a_{2}$ ) follows from

$$
\begin{aligned}
\sup _{n \in \mathbf{Z}}\left(\mathrm{E}\left\|\xi_{n}(m)-\xi_{n}\right\|_{X}^{2}\right)^{1 / 2} & \leq\|\xi(m)-\xi\|_{W}=\left\|\left(G_{m}^{-1}-G^{-1}\right) \eta\right\|_{W} \\
& \leq\left\|G^{-1}\left(I_{W}+G^{-1}\left(G_{m}-G\right)\right)^{-1}-G^{-1}\right\|_{L(W)}\|\eta\|_{W} \\
& \leq\left\|G^{-1}\right\|_{L(W)}\|\eta\|_{W} \gamma_{m}\left(1-\gamma_{m}\right)^{-1}=O\left(\delta_{m}\right), \quad m \rightarrow \infty .
\end{aligned}
$$

Theorem 2 is proved.

Remark 4. Results similar to Theorem 2 concerning the stability of stationary in a restricted sense solutions of the difference equation (2) with a finite numbers of nonzero operator coefficients are obtained by A. Ya. Dorogovtsev in [6, 7]. 
Remark 5. A unique mean square solution $\xi(m)$ of perturbed equation (13) corresponding to a stationary sequence $\eta$ is not necessarily stationary if $m \geq m_{0}$. One can only prove in this case that a solution of perturbed equations is mean square bounded (see [6, 17).

Remark 6. One can approximate a unique mean square bounded solution (13) for a fixed $m \geq m_{0}$ with successive approximations (see [5]). This method is used in [6, 7] to prove analogous results.

\section{BIBLIOGRAPHY}

1. M. F. Gorodniǔ, Solutions of a stochastic difference equation that are stationary and bounded in the mean, Dopov. Nats. Akad. Nauk Ukr. Mat. Prirodozn. Tekh. Nauki 8 (2002), 12-16. MR:2009511 (2004g:60099)

2. S. Bochner and R. S. Phillips, Absolutely convergent Fourier expansions for non-commutative normed rings, Ann. Math. 43 (1942), no. 3, 409-418. MR0007939(4:218g)

3. M. F. Gorodniı̌, Bounded and periodic solutions of a difference equation and its stochastic analogue in a Banach space, Ukrain. Mat. Zh. 43 (1991), no. 1, 41-46; English transl. in Ukrainian Math. J. 43 (1991), no. 1, 32-37. MR.1098269(92d:60068)

4. V. M. Kruglov, Supplementary Chapters on Probability Theory, "Vysshaya Shkola", Moscow, 1984. (Russian) MR756812 (86d:60009)

5. L. V. Kantorovich and G. P. Akilov, Functional Analysis, "Nauka", Moscow, 1984; English transl., Pergamon Press, Oxford-Elmsford, N.Y., 1982. MR664597 (83h:46002)

6. A. Ya. Dorogovtsev, Stability of periodic solutions of operator equations with perturbation coefficients, Exploring Stochastic Laws (A. V. Skorokhod and Yu. V. Borovskikh, eds.), VSP, Utrecht, The Netherlands, 1995, pp. 111-119. MR1713997 (2001g:60163)

7. A. Ya. Dorogovtsev, Stability of bounded and stationary solutions of linear equations with respect to perturbations of operator coefficients, Dokl. Akad. Nauk 345 (1995), no. 4, 448-450. MR.1376444 (96k:47123)

Department of Mathematical Analysis, Faculty for Mathematics and Mechanics, National Taras Shevchenko University, Glushkov Avenue, 6, Kyiv, 03127, Ukraine

E-mail address: gorodnii@yandex.ru

Received 18/MAR/2005

Translated by OLEG KLESOV 